\title{
De novo comparative transcriptome analysis of a rare cicada, with identification of candidate genes related to adaptation to a novel host plant and drier habitats
}

\author{
Zehai Hou and Cong Wei ${ }^{*}$
}

\begin{abstract}
Background: Although the importance of host plant chemistry in plant-insect interactions is widely recognized, our understanding about the genetic basis underlying the relationship between changes in midgut proteins and adaptation of plant-feeding insects to novel host plants and habitats is very limited. To address this knowledge gap, the transcriptional profiles of midguts among three populations of the cicada Subpsaltria yangi Chen were compared. Among which, the Hancheng $(\mathrm{HC})$ and Fengxiang $(\mathrm{FX})$ populations occurring in the Loess Plateau feed on Ziziphus jujuba Mill. var. spinosa (Bunge) Hu ex H. F. Chow, while the population occurring in a much drier habitat in the Helan $(\mathrm{HL})$ Mountains is locally specialized on a chemically divergent plant, Ephedra lepidosperma C. Y. Cheng.

Results: Based on comparative analysis, 1826 (HL vs HC) differentially expressed genes (DEGs) and 723 DEGs (HL vs FX) were identified between the populations utilizing different host plants, including 20,36, 2, 5 and 2 genes related to digestion, detoxification, oxidation-reduction, stress response and water-deprivation response, respectively, and 35 genes presumably associated with osmoregulation. However, only 183 DEGs were identified between the HC and FX populations, including two genes related to detoxification, two genes related to stress response, and one gene presumably associated with osmoregulation. These results suggest that the weakest expression differences were between the populations utilizing the same host plant and occurring in the closest habitats, which may help explain the metabolic mechanism of adaptation in S. yangi populations to novel host plants and new niches.
\end{abstract}

Conclusions: The observed differences in gene expression among $S$. yangi populations are consistent with the hypothesis that the host plant shift and habitat adaptation in the HL population was facilitated by differential regulation of genes related to digestion, detoxification, oxidation-reduction, stress response, water-deprivation response and osmoregulation. The results may inform future studies on the molecular mechanisms underlying the relationship between changes in midgut proteins and adaptation of herbivorous insects to novel host plants and new niches.

Keywords: Cicadidae, Midgut, Host shift, Host adaptation, Transcriptional variation, Plasticity, RNA-Seq

\section{Background}

For herbivorous insects, incorporation of a novel host into the diet and subsequent formation of distinct host associations play an important role in the early step of speciation process [1]. Plants produce a wide range of allelochemicals to defend against herbivore attack while,

\footnotetext{
* Correspondence: congwei@nwsuaf.edu.cn

State Key Laboratory of Crop Stress Biology for Arid Areas, and Key

Laboratory of Plant Protection Resources and Pest Management, Ministry of

Education, College of Plant Protection, Northwest A\&F University, Yangling

712100, Shaanxi, China
}

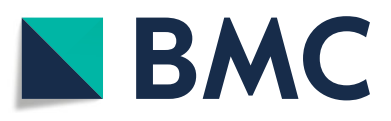

(c) The Author(s). 2019 Open Access This article is distributed under the terms of the Creative Commons Attribution 4.0 International License (http://creativecommons.org/licenses/by/4.0/), which permits unrestricted use, distribution, and

reproduction in any medium, provided you give appropriate credit to the original author(s) and the source, provide a link to the Creative Commons license, and indicate if changes were made. The Creative Commons Public Domain Dedication waiver (http://creativecommons.org/publicdomain/zero/1.0/) applies to the data made available in this article, unless otherwise stated.

in turn, insects have evolved different ways of coping with the chemical barriers. Due to the remarkable diversity of plant secondary compounds, an insect is inevitably confronted with different chemical environments when undergoing a host switch [2]. Consequently, host shift is an important driver of phenotypic and genetic changes for herbivorous insects [3]. Although the importance of insect adaptation to host chemistry has long been recognized, our understanding of the genetic basis of adaptation to new hosts is still insufficient [2]. 
Subpsaltria yangi Chen (Cicadidae: Tibicininae) is an endemic, Chinese cicada species [4-6], and male's intraspecific sexual mimicry in this species was revealed recently [7]. This rare species had been known to be distributed in the Loess Plateau and adjacent areas in Shaanxi Province and the Helan (hereafter HL) Mountains, Ningxia Hui Nationality Autonomous Region [4, 5, 7]. During the field investigations of S. yangi since 2011, a few more populations were discovered from the Loess Plateau and adjacent areas in Shaanxi, Shanxi and Gansu provinces [8]. We found that most populations of $S$. yangi occurring in the Loess Plateau and adjacent areas specialize on the sour jujube Ziziphus jujuba Mill. var. spinosa (Bunge) $\mathrm{Hu}$ ex $\mathrm{H}$. F. Chow (Rhamnaceae) (Fig. 1a). However, the population occurring in the Helan Mountains, surrounded by deserts or semi-deserts, utilizes Ephedra lepidosperma C. Y. Cheng (Ephedraceae) (Fig. 1b), although sympatric Z. jujuba var. spinosa is available (see Results). Genetic evidence and acoustic analysis of the calling song of males significantly indicate that the HL population is genetically differentiated from other populations of S. yangi, which provide a possible example of incipient speciation in insects [8]. This, coupled with the fact that $Z$. jujuba var. spinosa are abundant but not utilized as main host in the Helan Mountains, suggests that the population from the Helan Mountains has experienced a 'host shift'.

Identifying transcriptional changes associated with host shifts is an important step in understanding the molecular mechanisms of herbivorous insects adaptating to their new hosts $[2,9,10]$. Previous studies have demonstrated transcriptional regulations associated with insects feeding on different hosts. For example, research on host shift in a cactophilic fly, Drosophila mojavensis, has identified cytochrome P450s, glutathione S-transferases and UDP-glycosyltransferases as important gene classes likely involved in cactus utilization [11]. Research on host shift in another cactophilic fly, $D$. mettleri, revealed that host shift induces a number of genes involved in processes relevant to host adaptation, e.g., detoxification, central metabolism and chemosensory pathways [9]. De Panis et al. [12] suggested that transcriptome modulation during host shift is driven by secondary metabolites in the desert cactophilic fly D. buzzatii. Recently, Zhong et al. [10] suggested that transcriptional regulation of genes related to digestion, detoxifcation and ribosome may play an important role in adaptation of the striped stem borer Chilo suppressalis to a new host, the water-oat Zizania latifolia.

Cicadas are root pests feeding on the xylem fluids of their host plants and then emerge from the soil to use the tree canopies for mating, oviposition, and also additional feeding $[13,14]$. Populations of $S$. yangi feeding on different host plants may confront different chemical environments. The plant $Z$. jujuba var. spinosa is a thorny, deciduous plant widely distributed throughout the tropical and subtropical regions of the world [15]. The major compounds of branches and leaves of $Z$. jujuba var. spinosa are rutin, catechin, lupeol, quercetin-3-O- $\beta$-D-glucoside, zizyberanalic, epiceanothic and betulic acid $[16,17]$. In contrast, plants of the genus Ephedra are known to contain six types of alkaloids, i.e., ephedrine, pseudoephedrine, methylephedrine, methylpseudoephedrine, norephedrine and norpseudoephedrine, which have been used in traditional Chinese medicine for at least 5000 years [18]. Adult cicadas feed exclusively on the xylem fluid from branches of their host plants. Cicada nymphs are long lived underground and feed exclusively on the xylem sap from roots of their host plants. The cicada $S$. yangi may be a serious pest on the medicinal plant E. lepidosperma. Although the chemical composition in E. lepidosperma is not documented detailedly, this plant has been reported containing two types of alkaloids, ephedrine and pseudoephedrine [19]. Previous studies have shown that breeding in an alkaloid-rich host plant Trichocereus terscheckii $[20,21]$ affects fitness in D. buzzatii, such as decreasing viability, body size and starvation resistance, and extending development [22-25]. Therefore, adaptation to a new host plant such as $E$. lepidosperma for $S$. yangi entails the acquisition of mechanisms aimed to face such chemical challenges.
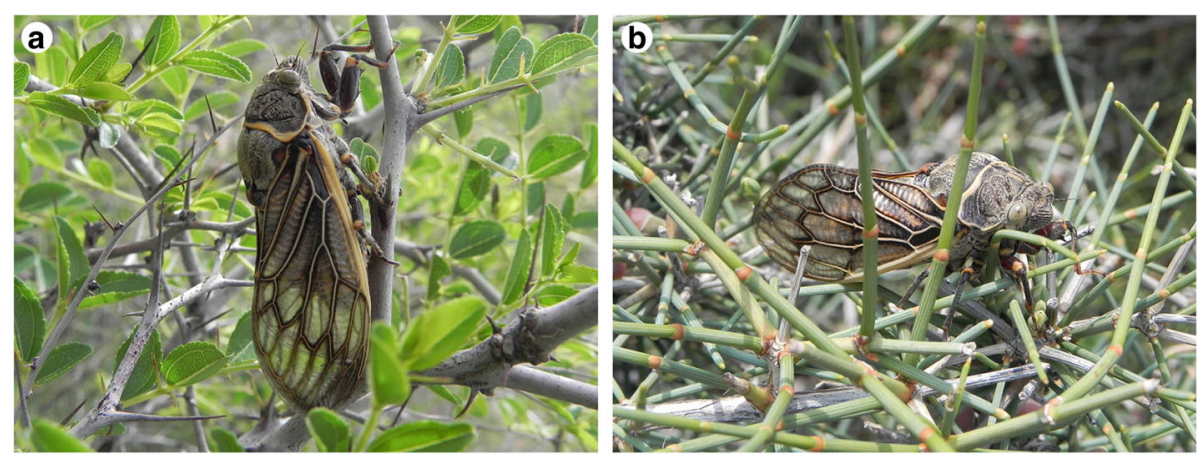

Fig. 1 Host plants of Subpsaltria yangi. a A S. yangi feeding on Ziziphus jujuba var. spinosa. b A S. yangi feeding on Ephedra lepidosperma. Both taken by Z. Hou 
This, coupled with geographic variation in host plant specialization, makes the $S$. yangi system especially amenable for studies on the genetic basis of new host adaptation.

Herbivorous insects show variability in their ability to use plants, which is based on differences in preference, ingestion speed, digestion efficiency, and detoxification $[26,27]$. Olfaction in antennae is essential for regulating insect behaviors such as host preference and oviposition site selection [28]. Many olfactory-related proteins are responsible for perceiving plant volatiles and mediating host selection of phytophagous insects $[29,30]$. Recently, Qi et al. [28] conducted transcriptome analysis based on RNA-Seq data from antennae of $S$. yangi to better understand the olfactory mechanisms in $S$. yangi which has a very narrow host range far fewer than diets of most other cicadas. They identified a number of olfactory genes of $S$. yangi, which provide direct evidence for future research of the olfactory system of $S$. yangi at the molecular level and provide information for elucidation of the molecular mechanisms and evolution of chemosensation in sap-sucking insects.

The midgut, the second largest organ in the insect body, actively interacts with the physical environment [31], which is the entry site for harmful bacteria, viruses and toxins, as well as for food and water [32]. The midgut also plays critical roles in digestion and nutrient uptake and, in particular, detoxification and oxidative stress responses. However, our understanding about the mechanisms underlying the relationship between changes in midgut proteins and adaptation of plant-feeding insects to different host plants is still very limited. Comparative studies are needed to elucidate mechanisms underlying the relationship between the changes in midgut proteins and the adaptation of insects to novel hosts. Thus, we compared gene expression in the midgut of different populations of $S$. yangi respectively feeding on $Z$. jujuba var. spinosa and E. lepidosperma. Our main goal was to detect differentially expressed genes (DEGs) in response to different host plants and habitats, and to test the hypothesis that the host plant shift in S. yangi is facilitated by differential regulation of genes related to digestion and detoxification. The results may also be helpful to explain the metabolic mechanism in S. yangi to adapt to new habitats and mechanisms of speciation in herbivorous insects.

\section{Results}

\section{Host plant utilization}

For the HL population, host plant utilizations of 402 and 345 individuals were recorded during the whole emergence period of 2016 and 2017, respectively. We found significantly more adults feeding on E. lepidosperma ( $N$ $=379)$ than on $Z$. jujuba var. spinosa $(N=23)$ (ratio =
379: $\left.23, x^{2}=313.50, P<0.001\right)$ in 2016 , and the individuals feeding on the former continued to predominate (ratio $=338: 7, x^{2}=315.65, P<0.001$ ) in 2017. The samples in 2016 and 2017 were pooled to obtain a significantly biased ratio toward to E. lepidosperma (ratio = 717: $\left.30, X^{2}=629.98, P<0.001\right)$. In contrast, our field investigation confirmed that the populations occurring in Hancheng (hereafter HC) and Fengxiang (hereafter FX) of Shaanxi Province are all feeding on Z. jujuba var. spinosa.

\section{Summary of sequences and assembly}

To quantify the gene expression patterns of midgut samples, we constructed nine cDNA libraries and then subjected them to deep sequencing using Illumina HiSeq2000. In total, $70.26 \mathrm{~Gb}$ high quality sequences were obtained from the transcriptome sequencing of midgut samples, ranging from 6.34 to $10.59 \mathrm{~Gb}$ per sample. The average error rates of the sequences are $0.01-0.02 \%$, and more than $91 \%$ of the bases with error rates $<0.1 \%$ (Additional file 1 : Table S1). The sequencing data were assembled into 418,777 transcripts with length ranging from 201 to 33,432 bases (mean length $=770$ bases, and median length $=368$ bases). As a result, 234,592 unigenes were obtained (mean length $=1149$ bases, and median length = 653 bases). This assembly had a high degree of completeness with a BUSCO score of $96.4 \%$, of which 693 (41.8\%) were complete single-copy BUSCOs, 906 (54.6\%) were complete duplicated BUSCOs, 27 (1.6\%) were fragmented BUSCOs, and $32(1.9 \%)$ were missing BUSCO orthologs out of the 1658 BUSCO groups searched.

\section{Functional annotation of the midgut unigenes}

In sum, 89,441 unigenes (38.1\% of the total unigenes) were annotated in at least one of the databases used in our study. Those unigenes were mostly annotated in the $\mathrm{Nr}$ database (30.9\%) (Additional file 1: Table S2). The highest percentage of unigene sequences were matched with Zootermopsis nevadensis (22.7\%), followed by Acyrthosiphon pisum (9.2\%), Lasius niger (5.7\%), Tribolium castaneum (4.6\%), and Diaphorina citri (4.5\%) (Additional file 1: Table S3).

Based on the Gene Ontology (GO) analysis, 59,179 (25.2\%) unigenes were mapped and clustered into cellular component, biological process and molecular function categories. The top two subcategories were: 1) 'cellular process' and 'metabolic process' in biological process category, 2) the 'cell' and 'cell part' in cellular component category, and 3) the 'binding' and 'catalytic activity' in molecular function category (Additional file 2: Figure S1). The results of Eukaryotic Orthologous Groups (KOG) analysis show that the matched 28,896 unigenes are divided into 26 categories. The dominant category includes 'general functional prediction only', 'signal transduction mechanisms', 
and 'posttranslational modification, protein turnover, chaperones' (Additional file 3: Figure S2). Kyoto Encyclopedia of Genes and Genomes (KEGG) analysis shows that the matched 22,621 (9.6\%) unigenes are assigned into 229 pathways, of which the top 32 are depicted in Additional file 4: Figure S3. The most well-represented metabolic pathways are involved in 'carbohydrate metabolism' (1383), 'lipid metabolism' (1133), 'amino acid metabolism' (958), respectively (Additional file 4: Figure S3).

\section{Identification of SSRs}

We detected 83,176 SSRs, and the most abundant type of SSR motifs are dinucleotide repeats mainly of (TG/ GT/AC/CA $)_{n}(48.8 \%$ ) (Fig. 2). About $38.5 \%$ of the SSRs are mononucleotide repeats mainly of $(\mathrm{A} / \mathrm{T})_{\mathrm{n}}$. Primers were successfully designed for 44,101 SSRs. For the purpose of molecular marker development, complex SSRs [e.g., (TTC $\left.)_{7}(\mathrm{TTA})_{7},(\mathrm{CG})_{6} \mathrm{catg}(\mathrm{CA})_{6}\right]$ and those with a motif unit size less than two nucleotides were removed. As a result, 18,670 SSR primers were obtained (Additional file 5: Table S4). The SSRs identified in our study may be useful for developing molecular markers to detect the genetic structure among populations of S. yangi.

\section{Differential gene expression analysis}

An overall view of gene expressions of midgut of $S$. yangi is presented in the hierarchical clustering heatmap (Additional file 6: Figure S4). The midgut sample of the $\mathrm{HC}$ population and that of the FX population cluster together, indicating a relatively high similarity in patterns of gene expression. Venn diagrams were used to summarize the number of differentially expressed genes (DEGs) among the three populations (Fig. 3a). First, we

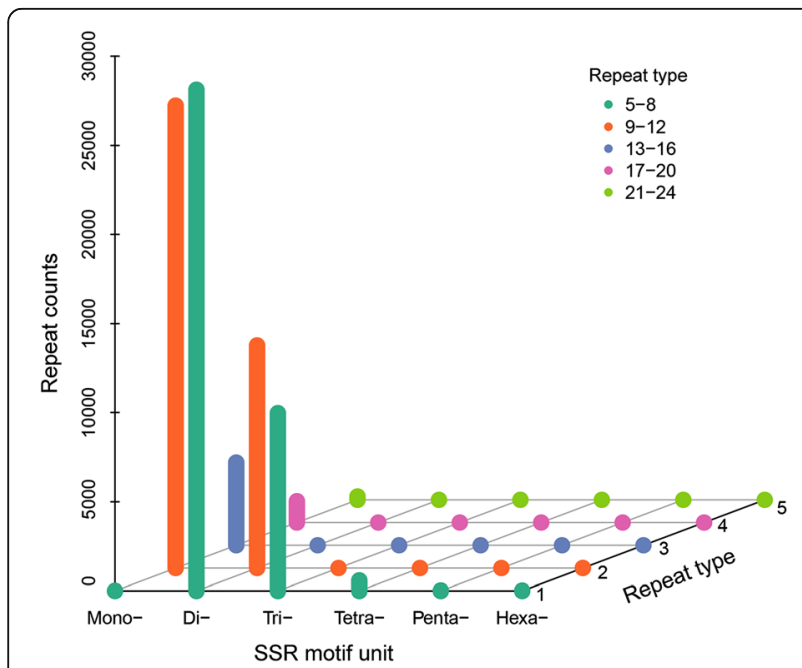

Fig. 2 Distribution of SSR motifs. The different color bars represent different repeat types (repeat number ranges of SSR motif unit)

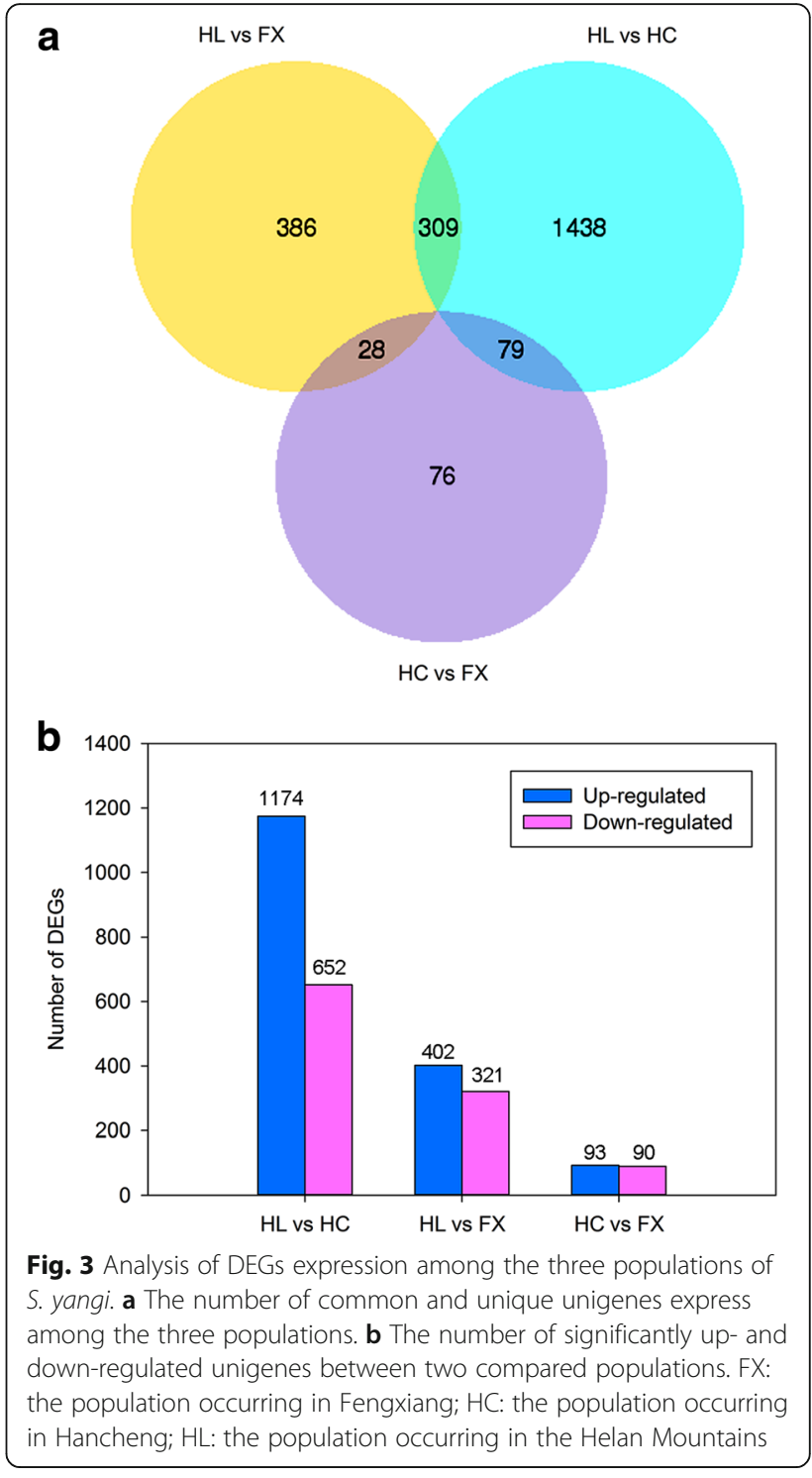

compared the expression profiles between $\mathrm{HC}$ (feeding on Z. jujuba var. spinosa) and FX (feeding on Z. jujuba var. spinosa), which represent the closest rearing condition in nature. It results in 183 DEGs identified in HC compared with FX, of which 93 were up-regulated and 90 were down-regulated (Fig. 3b; Additional file 7: Table S5). Second, we evaluated the differences in gene expression between the midgut samples of HL (feeding on E. lepidosperma) and HC, and detected 1826 DEGs, including 1174 up-regulated genes and 652 down-regulated genes (Fig. 3b; Additional file 8: Table S6). Then we analysed the differences in gene expression between HL and FX, and identified 723 DEGs, of which 402 were up-regulated and 321 were down-regulated (Fig. 3b; Additional file 9: Table S7). There are 309 DEGs shared between the latter two pairwise comparisons (Fig. 3a). 
In this study, we mainly focused on the transcriptional changes associated with a host plant shift in S. yangi. We identified 20 DEGs related to digestion in the comparative sets 'HL vs HC' and 'HL vs FX', encoding nine carbohydrases, five lipases and six proteases, and most of them were up-regulated in the HL population when compared with the HC and FX populations (Fig. 4; Additional file 10: Table S8). Thirty-five putative detoxification-related unigenes were also identified, including six cytochrome P450 monooxygenases (P450s), five UDP-glycosyltransferases (UGTs), six carboxylesterases (CEs), one glutathione S-transferase (GST) and 17 ATP-binding cassette (ABC) transporters (Fig. 5; Additional file 11: Table S9). Among which, most P450s and UGTs were down-regulated in the $\mathrm{HL}$ population when compared with the $\mathrm{HC}$ and FX populations. In addition to the 35 putative detoxificationrelated unigenes, we also identified an alkaline phosphatase, tissue-nonspecific isozyme-like gene (Cluster41,210.64298) exclusively expressed in the HL population (Fig. 5; Additional file 11: Table S9), indicating this gene may play a significant role in alkaloid resistance when $S$. yangi feeds on E. lepidosperma. We also found that two DEGs encoding peroxidase, i.e., one peroxidase (Cluster41,210.79117) and one peroxidase-like (Cluster-41,210. 159410), were up-regulated in the HL population when compared with the $\mathrm{HC}$ and FX populations (Fig. 5; Additional file 11: Table S9), which might play a role in protective response to oxidative stress, e.g., reactive oxygen species (ROS) ingested during feeding or food processing.

Besides coping with the host shift from $Z$. jujuba var. spinosa to E. lepidosperma, the HL population should at the same time be able to adapt to the habitats which are surrounded by deserts or semi-deserts. In our present study, we found that five unigenes encoding heat shock proteins (Hsp) were differentially expressed in the HL population when compared with the HC and FX populations, including one heat shock protein cognate 3 , one heat shock $70 \mathrm{kDa}$ protein cognate 4 , two heat shock 70 $\mathrm{kDa}$ protein cognate 5 and one heat shock protein $67 \mathrm{~B} 2$ (Fig. 6; Additional file 12: Table S10). We found that two DEGs encoding aquaporins, including one aquaporin AQPAe.a and one aquaporin 2-like protein, were up-regulated in the HL population when compared with the HC and FX populations (Fig. 6; Additional file 12: Table S10), suggesting that they may play an important role in response to water deprivation for the HL population which occurs in the desert or semi-desert environments of the Helan Mountains. In addition to Hsps and aquaporins, we also identified 35 DEGs encoding solute carriers (Slc), and majority of them were up-regulated in the HL population when compared with the $\mathrm{HC}$ and FX populations (Fig. 6; Additional file 12: Table S10).

However, using the same analytical procedures, we only identified five unigenes, including one UGT, one $A B C$ transporter, two Hsps and one Slc genes, differentially expressed in the comparative set ' $\mathrm{HC}$ vs $\mathrm{FX}$ ' (Table 1). These differences in gene expression suggest that the weakest expression differences were between the populations utilizing the same host plant and occurring in the closest habitats. These observed differences in gene expression between populations utilizing different host plants and occurring in different habitats may help explain the metabolic mechanism of adaptation in $S$. yangi populations to novel host plants and new niches.

\section{Mean fixation index values of individual genes}

Mean fixation index (Fst) values of individual genes varied broadly in comparisons between populations occurring in

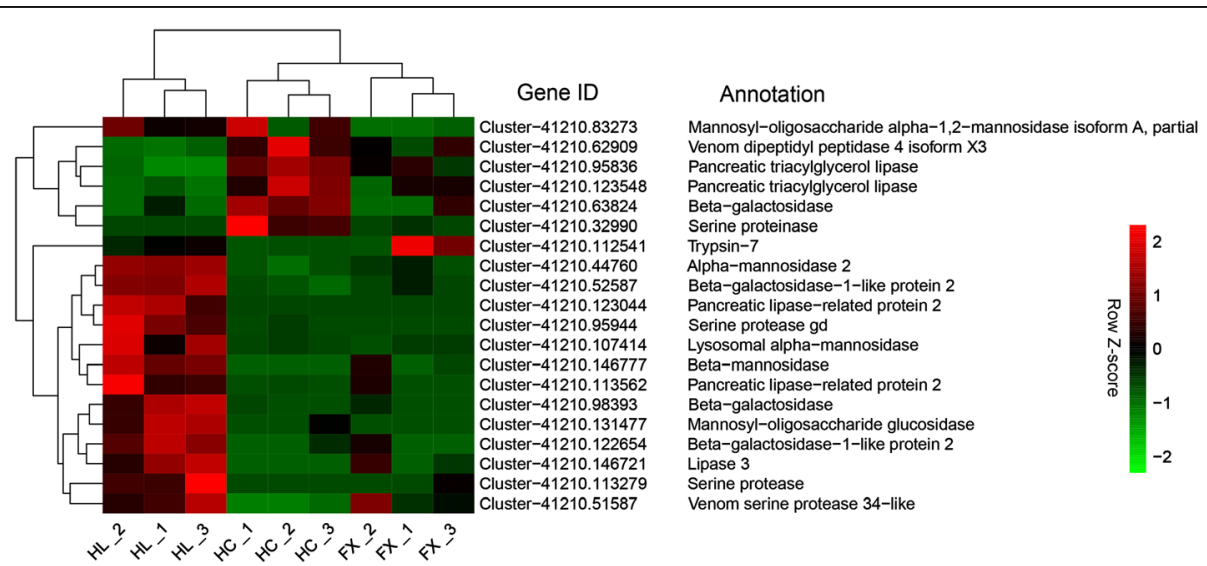

Fig. 4 Heatmap of normalized FPKM of DEGs related to digestion. The Z-score represents the deviation from the mean by standard deviation units. Red color indicates up-regulated expression, whereas green color indicates down-regulated expression. FPKM: fragments per kilobase of transcript per million fragments mapped; FX: the population occurring in Fengxiang; HC: the population occurring in Hancheng; HL: the population occurring in the Helan Mountains 


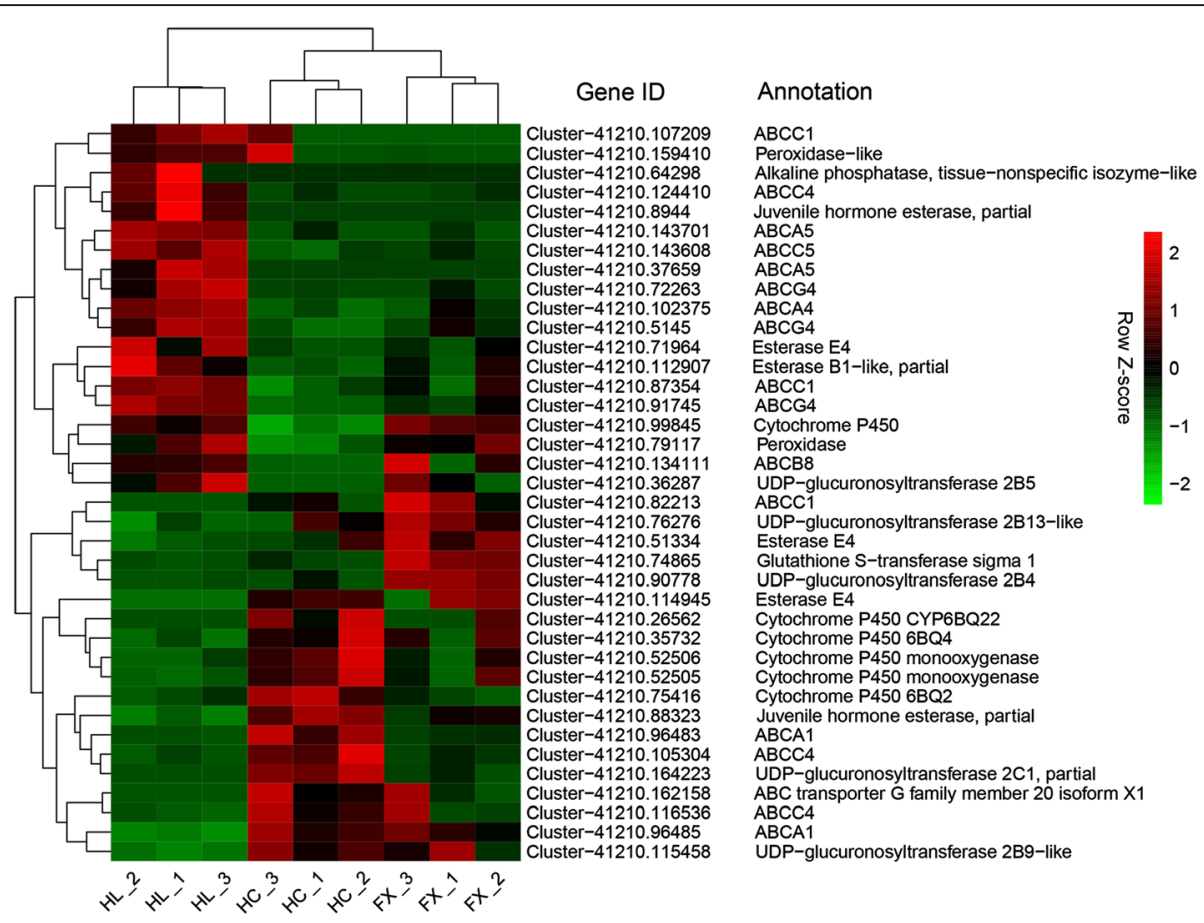

Fig. 5 Heatmap of normalized FPKM of DEGs related to detoxification and oxidation-reduction. The Z-score represents the deviation from the mean by standard deviation units. Red color indicates up-regulated expression, whereas green color indicates down-regulated expression. FPKM: fragments per kilobase of transcript per million fragments mapped; FX: the population occurring in Fengxiang; HC: the population occurring in Hancheng; HL: the population occurring in the Helan Mountains

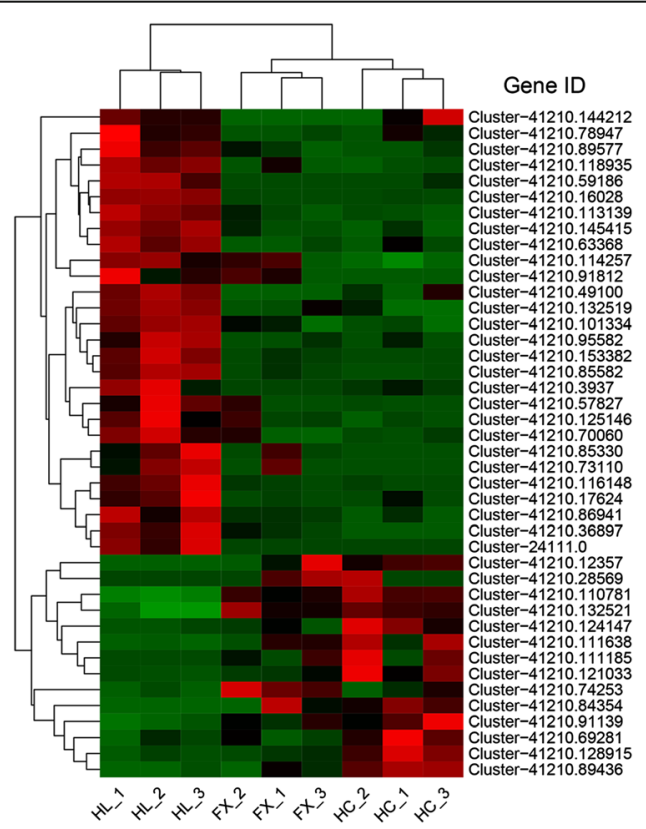

Annotation

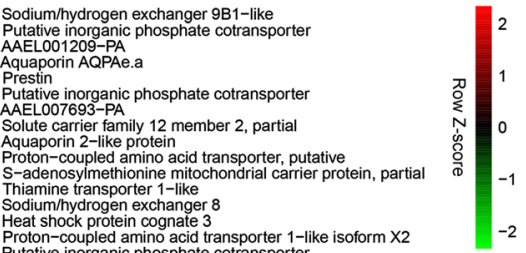

Protive inpled amino acid transporter 1 -like isoform $\times 2$ Heat shock $70 \mathrm{kDa}$ protein cognate 5

Heat shock $70 \mathrm{kDa}$ protein cognate 4

Zinc transporter 9

Zinc transporter foi

Solute carrier family 23 member 2 -like
Solute carrier family 23 member 2-like

(1)

Mitochonondial thiamine pyrophosphate carrier-like
Solute carrier organic anion transporter family member 2A1-like

Solute carrier organic anion transporter family member $2 \mathrm{~A} 1$-lik
Solute carrier family 35 member B1-like protein

Sodium-independent sulfate anion transporter-like isoform $X$

Sodium-independent sulfate anion transporter-like iso
Sodium-coupled monocarboxylate transporter 1-like

Prestin Sugar transporter ERD6-like 4

Zinc transporter ZIP14

Solute carrier family 25 member 36

Solute carrier family 12 member 9

Solute carrier family 12 member 9
Heat shock protein 6732
Heat shock 70 KDa protein cognate 5

Zinc transporter 1
Solute carrier family 2 , facilitated glucose

Sodium- and chloride-dependent transporter XTRP3

Fig. 6 Heatmap of normalized FPKM of DEGs related to stress response, water-deprivation response and putative osmoregulation. The Z-score represents the deviation from the mean by standard deviation units. Red color indicates up-regulated expression, whereas green color indicates down-regulated expression. FPKM: fragments per kilobase of transcript per million fragments mapped; FX: the population occurring in Fengxiang; $\mathrm{HC}$ : the population occurring in Hancheng; HL: the population occurring in the Helan Mountains 
Table 1 Differentially expressed genes identified in the comparative set 'HC vs FX'

\begin{tabular}{llll}
\hline Gene ID & Annotation & $\log _{2}$ FC (HC vs FX) & $q$ value \\
\hline Cluster-41,210.79881 & UDP-glucuronosyltransferase 2B4 & $+\infty$ & $2.31 \mathrm{E}-05$ \\
Cluster-36,972.0 & ABC transporter G family member 23 & $-\infty$ & $3.25 \mathrm{E}-02$ \\
Cluster-41,210.118981 & Heat shock 70 kDa protein cognate 4 & $+\infty$ & $5.39 \mathrm{E}-04$ \\
Cluster-41,210.165451 & 30 kDa heat shock protein & $+\infty$ & $3.08 \mathrm{E}-03$ \\
Cluster-41,210.137221 & Sodium-dependent phosphate transporter 2 & 7.58 & $8.05 \mathrm{E}-03$
\end{tabular}

$F C$ fold change, $F X$ the population occurring in Fengxiang, $H C$ the population occurring in Hancheng; $+\infty$ : genes exclusively expressed in the $H C$ population; $-\infty$ : genes exclusively expressed in the FX population

different habitats and utilizing different host plants. Mean Fst values of venom serine protease 34-like gene and solute carrier family 12 member 2, partial gene between HL and FX are 0.08 and 0.11 , respectively, indicating a moderate level of genetic differentiation (Table 2). Mean Fst value of $A B C C 4$ gene between $\mathrm{HL}$ and $\mathrm{HC}$ is 0.16 (Table 3), also indicating a moderate level of genetic differentiation. While mean Fst values of heat shock protein cognate 3 gene and heat shock protein $67 B 2$ gene between HL and FX are 0.47 and 1 , respectively (Table 2), indicating a high level of genetic differentiation. Mean Fst values of venom serine protease 34-like gene, cytochrome P450 6BQ4 gene, solute carrier family 12 member 2, partial gene, heat shock protein cognate 3 gene and heat shock protein $67 B 2$ gene between $\mathrm{HL}$ and $\mathrm{HC}$ are $0.45,0.57,0.63,0.80$ and 0.25 , respectively (Table 3 ), also indicating a high level of genetic differentiation.

\section{Enrichment pathway analysis of DEGs}

We mapped all the DEGs to terms in the KEGG database. KEGG pathways with $P$-value $<0.05$ between the two pairwise comparisons, i.e., ' $\mathrm{HL}$ vs $\mathrm{HC}$ ' and ' $\mathrm{HL}$ vs FX', are provided in Additional file 1: Table S11. In the comparison of 'HL vs $\mathrm{HC}$, 'ABC transporters' and 'pancreatic secretion' pathways were up-regulated, while 'citrate cycle (TCA cycle), 'fat digestion and absorption', and 'insulin resistance' were down-regulated. In the comparison of 'HL vs FX', 'ABC transporters' were up-regulated, while 'insulin signaling pathway,' 'glucagon signaling pathway' and 'vitamin digestion and absorption' were down-regulated.

In both pairwise comparisons of ' $\mathrm{HL}$ vs $\mathrm{HC}$ ' and ' $\mathrm{HL}$ vs FX', the strongest changes in the top $20 \mathrm{GO}$ categories were 'tRNA (m1A) methyltransferase complex', 'methyltransferase complex,' 'tRNA methyltransferase complex' of cellular component (CC) and 'tRNA methylation' of biological process (BP) (Additional file 13: Figure S5). These four changes were significant enriched, with $-\log _{2}(P$-value) higher than 16 in 'HL vs FX'. Among the top 20 of the strongest GO categories, eleven were common in 'HL vs HC' and 'HL vs FX' (Additional file 13: Figure S5).

\section{Validation of transcriptome data using $\mathrm{qPCR}$}

To validate the RNA-seq results, the relative expression levels of 15 selected genes were analysed by RT-qPCR, including 12 DEGs in 'HL vs HC' and three DEGs in 'HL vs FX'. Among these 15 different expression genes, the majority exhibit a consistent expression pattern between RNA-Seq and qPCR (Fig. 7), indicating that RNA-seq data are reliable.

Table 2 Population genetics and expression data for the genes in the comparative set 'HL vs FX'

\begin{tabular}{|c|c|c|c|c|c|c|}
\hline Gene ID & Nr Description & Tajima's D HL & Tajima's D FX & Mean Fst & $\log _{2} F C(H L$ vs $F X)$ & $q$ value \\
\hline Cluster-41,210.51587 & Venom serine protease 34 -like & 1.75 & 0.31 & 0.08 & 0.42 & 1 \\
\hline Cluster-41,210.75416 & Cytochrome P450 6BQ2 & 0.96 & 1.97 & -0.23 & -0.35 & 1 \\
\hline Cluster-41,210.35732 & Cytochrome P450 6BQ4 & 0 & -0.05 & $5.55 \mathrm{E}-17$ & -1.98 & $9.98 \mathrm{E}-01$ \\
\hline Cluster-41,210.51334 & Esterase E4 & NA & 0 & -0.096 & -3.41 & $1.63 \mathrm{E}-02$ \\
\hline Cluster-41,210.96485 & $A B C A 1$ & NA & 0 & $-1.14 \mathrm{E}-16$ & -2.76 & $7.54 \mathrm{E}-02$ \\
\hline Cluster-41,210.105304 & $\mathrm{ABCC} 4$ & 0 & -1.13 & -0.12 & -1.03 & 1 \\
\hline Cluster-41,210.91139 & Zinc transporter 1 & NA & 0 & -0.096 & -3.0 & $1.31 \mathrm{E}-01$ \\
\hline Cluster-41,210.111638 & Solute carrier family 25 member 36 & 0 & 0 & -0.29 & -4.91 & 2.54E-01 \\
\hline Cluster-41,210.145415 & Solute carrier family 12 member 2 , partial & NA & -0.19 & 0.11 & 2.28 & 3.10E-01 \\
\hline Cluster-41,210.101334 & Heat shock protein cognate 3 & -1.13 & 0 & 0.47 & 1.73 & $9.51 \mathrm{E}-01$ \\
\hline Cluster-41,210.74253 & Heat shock protein 67B2 & NA & NA & 1 & -5.04 & 3.57E-03 \\
\hline
\end{tabular}

$F C$ fold change, $F X$ the population occurring in Fengxiang, $H L$ the population occurring in the Helan Mountains, NA Not available 
Table 3 Population genetics and expression data for the genes in the comparative set ' $\mathrm{HL}$ vs HC'

\begin{tabular}{|c|c|c|c|c|c|c|}
\hline Gene ID & Nr Description & Tajima's D HL & Tajima's D HC & Mean Fst & $\log _{2} \mathrm{FC}(\mathrm{HL}$ vs HC) & $q$ value \\
\hline Cluster-41,210.51587 & Venom serine protease 34 -like & 1.75 & NA & 0.45 & 3.76 & $6.58 \mathrm{E}-04$ \\
\hline Cluster-41,210.75416 & Cytochrome P450 6BQ2 & 0.96 & 1.25 & -0.13 & -2.92 & 1.33E-02 \\
\hline Cluster-41,210.35732 & Cytochrome P450 6BQ4 & 0 & 0 & 0.57 & -2.71 & 4.55E-02 \\
\hline Cluster-41,210.105304 & $\mathrm{ABCC} 4$ & 0 & 0 & 0.16 & -3.12 & 3.93E-03 \\
\hline Cluster-41,210.111638 & Solute carrier family 25 member 36 & 0 & 0 & -0.50 & -6.11 & 5.49E-03 \\
\hline Cluster-41,210.145415 & Solute carrier family 12 member 2 , partial & NA & 1.03 & 0.63 & 2.75 & 7.47E-03 \\
\hline Cluster-41,210.101334 & Heat shock protein cognate 3 & -1.13 & NA & 0.80 & 2.91 & $5.26 \mathrm{E}-03$ \\
\hline Cluster-41,210.74253 & Heat shock protein 67B2 & NA & 0 & 0.25 & -3.25 & 1 \\
\hline Cluster-41,210.3937 & Heat shock $70 \mathrm{kDa}$ protein cognate 4 & NA & 0 & 8.33E-17 & 2.59 & 0.45 \\
\hline
\end{tabular}

FC fold change, $H C$ the population occurring in Hancheng, $H L$ the population occurring in the Helan Mountains, NA Not available
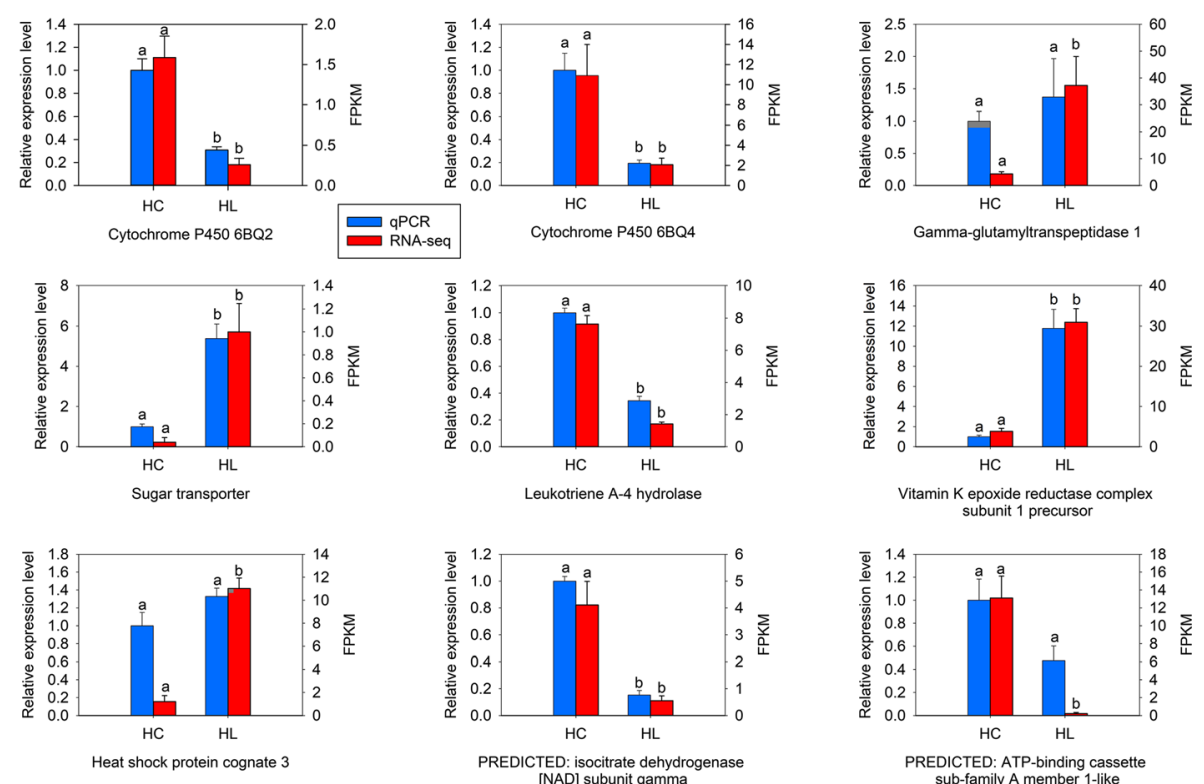

Heat shock protein cognate 3

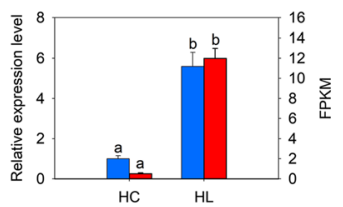

Hypothetical protein Y032_0002g724

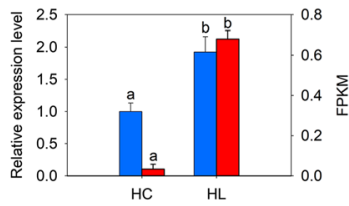

Hypothetical protein M514_21119
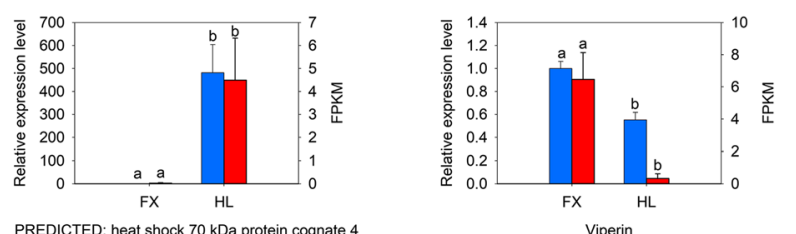

sub-family A member 1-like

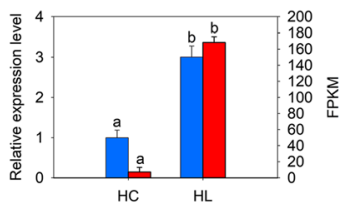

Hypothetical protein BRAFLDRAFT_90379

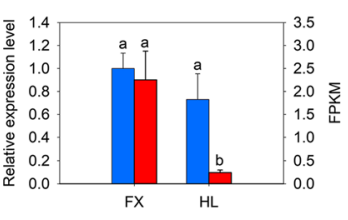

Thyroid peroxidase precursor, putative

Fig. 7 Gene expression levels of differentially expressed genes through RNA-seq and qRT-PCR analysis. FPKM: fragments per kilobase of transcript per million fragments mapped; FX: the population occurring in Fengxiang; HC: the population occurring in Hancheng; HL: the population occurring in the Helan Mountains 


\section{Discussion}

RNA-Seq is revolutionizing the fields of ecological and evolutionary genomics, and contributing new insights into the responses that organisms deploy in alternative environments (e.g., plant hosts) [12]. This approach has been employed to evaluate the genetic changes, from a broad view and at the level of candidate genes, in response to different host plants in insects $[9,11,12,33,34]$. In the present study, the strongest gene expression differences were revealed in the pairwise comparison between 'HL vs HC' (1826 DEGs), followed by the comparison between 'HL vs FX' (723 DEGs), while the weakest expression differences were found in the comparison between ' $\mathrm{HC}$ vs FX' (183 DEGs) (Fig. 3a). This is in line with the genetic differentiation among populations of $S$. yangi based on mitochondrial $(\mathrm{COI}+\mathrm{COII}+\mathrm{Cytb}+\mathrm{A6A8})$ genes, where the mean Fst among the three comparisons, 'HL vs HC', 'HL vs FX' and ' $\mathrm{HC}$ vs FX' was $0.0735,0.0676$ and 0.0121 , respectively [8]. This suggests that differential gene expression in the midgut among these populations is closely associated with genetic variations of related populations.

The HC and FX populations of S. yangi specialize on $Z$. jujuba var. spinosa, while the HL population has shifted to chemically divergent E. lepidosperma. The two host plants differ in biochemical composition and, thus, are expected to have a different nutritional value for $S$. yangi. Our results show that the genes related to digestion were mainly up-regulated in the HL population when compared with the HC and FX populations (Fig. 4). Our results might indicate that the DEGs related to digestion were involved in adaptation to host shift in $S$. yangi.

Some differentially expressed metabolism-related genes of insects in adapting to different biochemical composition of host plants have been revealed [9, 12, 34]. For example, Chikate et al. [35] chose a set of trypsin and chymotrypsin transcripts and inspected their pattern of expression of the cotton bollworm Helicoverpa armigera in response to host plants with different nutritional qualities. It is suggested that serine proteases are key enzymes allowing this lepidopteran pest to adapt to many different host plants and, therefore, augmenting polyphagy [35]. Celorio-Mancera et al. [33] reached similar conclusions in the butterfly larvae of Polygonia c-album. In our present study, we also reveal that four genes (Cluster-41,210.32990, Cluster-41,210.95944, Cluster-41,210.113279, Cluster-41,210.51587) encoding serine proteases were differentially expressed in the $\mathrm{HL}$ population when compared with the HC and FX populations (Fig. 4; Additional file 10: Table S8), which may be involved in host selection and adaptation of S. yangi.

Besides the nutritional requirements, insects should at the same time be able to cope with toxic chemicals from their host plants. When confronted with host shift, insects can successfully survive on their new host plant through enzymes coded by detoxifying genes such as P450s, GSTs, UGTs and CEs [9, 11, 12, 33, 34]. From the midgut of S. yangi, besides P450s, we also found that five UGTs, six CEs and one GST were differentially expressed in the HL population when compared with the $\mathrm{HC}$ and FX populations. In addition to detoxifying enzymes, $\mathrm{ABC}$ transporters are also involved in detoxification [36], which are membrane-bound proteins involved in the movement of various substrates, including drugs and insecticides, across the lipid membrane [37]. The $A B C$ superfamily is currently divided into eight distinct subfamilies $(\mathrm{A}-\mathrm{H})$ [38]. Among which, three subfamilies, $\mathrm{ABCB}, \mathrm{ABCC}$ and $\mathrm{ABCG}$, are involved in drug resistance [39]. In our study, differentially expressed $A B C$ transporters were also detected, including $\mathrm{ABCB} 8, \mathrm{ABCC} 1$, $A B C C 4, A B C C 5$ and $A B C G 4$, and majority of them were up-regulated in the HL population when compared with the HC and FX populations (Fig. 5; Additional file 11: Table S9). This suggests that these genes may play an important role in detoxification during the host plant shift of this cicada species. Besides the abovementioned proteins that are involved in detoxification, alkaline phosphatase is also associated with removal of alkaloids in pests, and high levels of alkaline phosphatase have been implicated in insecticide resistance $[40,41]$. In our study, we found that an alkaline phosphatase, tissue-nonspecific isozyme-like gene (Cluster-41,210.64298) was exclusively expressed in the HL population (Fig. 5; Additional file 11: Table S9), indicating this gene may play a significant role in alkaloid resistance when S. yangi feeds on E. lepidosperma.

Reactive oxygen species (ROS) are derived from the metabolism of molecular oxygen [42], such as superoxide radicals $\left(\mathrm{O}_{2}{ }^{-}\right)$, hydroxyl radical $\left(\mathrm{OH}^{-}\right), \mathrm{H}_{2} \mathrm{O}_{2}$, and hydroperoxides $(\mathrm{ROOH})$, which are generated by exogenous and endogenous sources [43]. Exogenous sources, for example prooxidant allelochemicals produced by host plants, pose a serious challenge to herbivorous insects during insect-plant interactions. However, some herbivorous insects have evolved a midgut-based defense against the toxic effects of ROS [44, 45]. They can produce many enzymes for detoxifying ROS, including glutathione peroxidase (GPX), catalase (CAT), superoxide dismutase (SOD) and ascorbate peroxidase [46]. Mittapalli et al. [44] suggested that the induced expression of antioxidant genes, such as phospholipid glutathione peroxidases, catalases and superoxide dismutases in the midgut of a major insect pest of wheat worldwide, the Hessian fly Mayetiola destructor, to be a protective response to ROS ingested during feeding or food processing. Zhu et al. [47] also suggested that peroxidase potentially involved in insecticide metabolic resistance in the diamondback moth Plutella xylostella. In our present study, two DEGs encoding peroxidase were identified between 
the populations of $S$. yangi utilizing different host plants (Fig. 6; Additional file 12: Table S10), suggesting that they may also play an important role in protective response to ROS ingested during feeding or food processing.

When organisms are exposed to heat, cold or some other stresses, they can synthesize Hsps which participate in unfolding and relocalization of proteins damaged by the stresses [48]. Although a number of investigations have confirmed the importance of Hsps in resistance towards heat, cold and a range of other stresses, e.g., insecticides, heavy metals, desiccation, diseases, parasites and inbreeding, the most important role of Hsps is in response to stress resistance and adaptation to the environment [49]. However, Hsps expression confers some costs to fertility/fecundity, energy, development and survival $[50,51]$. Therefore, organisms do not express Hsps unless environmental temperatures become severe, and their ability to induce Hsps is usually related to temperature conditions in their habitats [52]. For example, the limpet Collisella scabra inhabiting the high intertidal region has a greater tolerance to high temperatures and produces Hsps at a higher temperature than the related species C. pelta inhabiting the more sheltered upper midtidal region [53]. Some species of the freshwater coelenterate hydra Hydra from stable environments lack an ability to induce Hsps, while other related species from fluctuating environments exhibit heat shock response [54]. In addition, Bosch et al. [54] suggested that the ability of organisms to live in stressful habitats might be related to the ability to produce Hsps in response to stress, and that different organisms may utilize the same Hsp in different manners or produce different Hsps in response to thermal stress in different habitats. In our present study, we found that five unigenes encoding Hsps were differentially expressed in the HL population when compared with the $\mathrm{HC}$ and FX populations (Fig. 6; Additional file 12: Table S10). The habitats of the HL population, surrounded by deserts or semi-deserts, occupy at higher latitude and higher altitude than that of the $\mathrm{HC}$ and FX populations (Table 4). Hsps may play an important role in response to temperature stress and adaptation to the desert or semi-desert environments in the HL population of S. yangi.

Animals living in desert environments are forced to endure intense heat and drought, and as a result, species living in deserts likely involves a large number of adaptive traits, e.g., the ability to maintain the proper water and solute balance $[55,56]$. Aquaporins (AQP), a large protein family facilitate transport of water between cells [57] and maintain water balance in the kidney [58-60]. For example, AQP2 transports water back into the kidney from the collecting duct $[61,62]$ and plays an essential role in the urinary concentrating mechanism in mammals. Mice lacking AQP2 suffer severe polyuria, with average basal daily urine volumes approximately equivalent to body weight and are totally unable to concentrate urine [63]. Similarly, mice lacking AQP1 and AQP4 also presented defects in water reabsorption [63]. While AQP4 is apparently absent in desert species such as kangaroo rats [64] and camelids [65]. In our study, an aquaporin AQPAe.a gene (Cluster-41,210.118935) and an aquaporin 2-like gene (Cluster-41,210.63368) were up-regulated in the HL population when compared with the HC and FX populations (Fig. 6; Additional file 12: Table S10). This suggests that they may play an important role in response to water deprivation for the HL population which occurs in the desert or semi-desert environments of the Helan Mountains. Regarding solute balance, another gene family, solute carriers (Slc), are responsible for the maintenance of electrolyte levels $[66,67]$. Several studies have revealed that some members of solute carriers play a significant role in the osmoregulation in desert animals [56, 68-70]. For example, solute carrier family 2 member 9 (Slc2a9) has been suggested to be undergoing positive selection in kidney transcriptomes in the desert-adapted mouse Peromyscus eremicus [69]. A study of differential gene expression in the cactus mouse $P$. eremicus revealed that Slc2a1 and Slc8a1 showed responses to acute dehydration [56]. Recently, Giorello et al. [71] considered Slc12a2 may be related to water conservation in desert animals. In our present study, a unigene encoding Slc12a2 was up-regulated in the HL population (Fig. 6; Additional file 12: Table S10), suggesting that it may also be related to water conservation in the desert or semi-desert environments.

Host shift and subsequent adaptation can drive evolution and diversification of plant-feeding insects $[9,72]$. In the context of a host switch, transcriptional plasticity in metabolic pathways involved in processing and detoxifying food resources may produce a phenotype that is well matched to the new environment (e.g., the deserts

Table 4 Host plants and locality data of S. yangi investigated in this study

\begin{tabular}{|c|c|c|c|c|c|}
\hline Population & Location & Host plant & Latitude & Longitude & Elevation (m) \\
\hline$\overline{\mathrm{HL}}$ & $\begin{array}{l}\text { The Helan (HL) Mountains, } \\
\text { Ningxia Hui Nationality } \\
\text { Autonomous Region }\end{array}$ & Ephedra lepidosperma C. Y. Cheng & $38^{\circ} 33.699^{\prime} \mathrm{N}$ & $105^{\circ} 55.217^{\prime} \mathrm{E}$ & $1400-1600$ \\
\hline $\mathrm{HC}$ & Hancheng (HC), Shaanxi & $\begin{array}{l}\text { Ziziphus jujuba Mill. var. spinosa (Bunge) } \\
\text { Hu ex H. F. Chow }\end{array}$ & $35^{\circ} 31.683^{\prime} \mathrm{N}$ & $110^{\circ} 25.927^{\prime} \mathrm{E}$ & 582 \\
\hline FX & Fengxiang (FX), Shaanxi & Z. jujuba var. spinosa & $34^{\circ} 31.273^{\prime} \mathrm{N}$ & $107^{\circ} 24.044^{\prime} \mathrm{E}$ & 800 \\
\hline
\end{tabular}


or semi-deserts). Directional selection is expected to further alter the plastic response in the direction of the optimum, resulting in adaptive evolution (known as the Baldwin effect) [73]. In this case, the DEGs detected in the midgut of $S$. yangi might be initially beneficial in the process of host shift in this species, which may subsequently evolve in a long-term feeding on a new host plant (viz., E. lepidosperma). A previous study indicated that the HL population has evolved into a distinct independent lineage according to the analysis of population differentiation based on both molecular data and males' calling song structure [8]. The deserts or semi-deserts surrounding the Helan Mountains were previously shown as a major dispersal and climatic barrier for gene flow of related animals [74]. Such barriers appear to have promoted divergence of the HL lineage from other lineages of S. yangi [8]. Separated populations are potentially to become distinct species, as separated populations are seldom to get reunited after long-term chance and selection reciprocity, which may cause such populations to diverge and enhance many traits associated with reproductive isolation [75]. Differences in the patterns of gene expression in pairwise comparisons of ' $\mathrm{HL}$ vs $\mathrm{HC}$ ' and 'HL vs FX' may be representative of the genetic changes which facilitate the colonization of novel habitats for $S$. yangi, and help to mitigate the stresses imposed by a novel host containing different xenobiotics.

Differential gene expression could be affected by several factors, such as genetic variations between populations [71], response to different host plants $[9,11,12]$, response to abiotic factors (i.e., temperature) [76-78], or response to different microbiota in the alimentary canal [79-82]. Our analysis revealed that a certain number of genes were differentially expressed among populations utilizing different host plants and living in different habitats. Furthermore, the weakest expression differences were found between the populations utilizing the same host plant and occurring in the closest habitats. Given that the HL population is locally specialized on the endemic host plant $E$. lepidosperma but other populations specialize on $Z$. jujuba var. spinosa, results of our present study suggest that host shift could, coupled with geographical barriers (i.e., the deserts or semi-deserts surrounding the Helan Mountains) and ecological changes, have acted as effective factors resulting in the population divergence and allopatric speciation in S. yangi. Further study is required to confirm that differential expression of the various DEGs identified in our comparative analysis of cicada transcriptomes is due to the mechanisms of adaptation to the novel host plant and new habitats.

\section{Conclusions}

In this study, we investigated the transcriptional changes in the midgut of the cicada Subpsaltria yangi. Our results indicate that the DEGs related to digestion, detoxification, oxidation-reduction, stress response, waterdeprivation response and osmoregulation may be important in the adaptation to host plants and habitats by S. yangi. The observed differences in gene expression among S. yangi populations occurring in different habitats and utilizing different host plants may help explain the metabolic mechanism of adaptation in S. yangi populations to a novel host plant and drier habitats, and inform future studies on the molecular mechanisms of population divergence or speciation in herbivorous insects.

\section{Methods}

Field investigation of host plant utilization by Subpsaltria yangi

The host plant utilization by $S$. yangi was investigated during its summer emergence period. Specifically, during the behavioral ecology study of the population occurring in the Helan Mountains of Ningxia Hui Nationality Autonomous Region from May to June, 2016 and 2017, virgin adults of S. yangi were captured at night or in the early morning when they emerged from the soil. We simultaneously recorded what host plant the insects fed on in habitats of the Helan Mountains where Z. jujuba var. spinosa and E. lepidosperma are both available. For the populations from Hancheng and Fengxiang, Shaanxi Province, the host plant utilization of adults was investigated during specimen collection at the same season in 2016 and 2017.

\section{Insect collection and dissection}

Transferring cicada nymphs to new roots is a difficult process and results in high mortality [83, 84]. Adult cicadas are also difficult to rear in the laboratory. Thus, adults collected from wild populations were used in this study. The plant E. lepidosperma is an endemic species to the Helan Mountains [19]. It is very common that many habitats in the Helan Mountains only contain the host plant E. lepidosperma for S. yangi, although the host plant $Z$. jujuba var. spinosa mixes with the former in a few habitats. To obtain representative S. yangi samples exclusively feeding on E. lepidosperma, S. yangi adults from the Helan Mountains were collected at the habitats that only contain E. lepidosperma in June, 2016. In order to obtain representative S. yangi samples exclusively feeding on $Z$. jujuba var. spinosa, adults from Hancheng feeding on Z. jujuba var. spinosa were collected in June, 2016. Due to the less controlled conditions of the two populations, another population feeding on $Z$. jujuba var. spinosa in Fengxiang that has a similar habitat to the $\mathrm{HC}$ population was also used to strengthen the results, which was collected in June, 2016 (Table 4). 
Collected adults from the three localities were reared on their corresponding host plants and transferred alive to the laboratory for dissection and RNA extraction. Midguts of the adults were dissected on ice in sterile phosphate buffered saline (PBS) treated with $0.1 \%$ diethylpyrocarbonate (DEPC) under an Olympus SZX 10 stereomicroscope (Olympus Corporation, Tokyo, Japan), and washed in sterile ice-cold PBS to remove the sap of host plants, then were immediately frozen and stored at $-80^{\circ} \mathrm{C}$ for subsequent RNA extraction.

\section{RNA extraction, cDNA library preparation and Illumina sequencing}

For transcriptome sequencing, one sample included the midgut from one adult of $S$. yangi was collected. Total RNA was extracted with Trizol reagent (Life Technologies, USA) according to the manufacturer's instructions. DNA contaminants were removed by treating RNA extraction products with RNase-free DNase (Ambion, Austin, TX, USA), and then were purified through phenol-chloroform extraction. RNA quality was examined using $1 \%$ agarose gel and the concentration was determined using NanoDrop (Thermo). In total, nine RNA samples ( 3 populations $\times 3$ replicated samples) were obtained. Sequencing libraries were constructed using NEBNext ${ }^{\circ}$ Ultra $^{\text {Tu }}$ RNA Library Prep Kit for Illumina ${ }^{\circ}$ (NEB, USA) following manufacturer's recommendations, and index codes were added to attribute sequences to each sample. In brief, mRNA was purified from total RNA using poly-T oligo-attached magnetic beads and then was fragmented. Double-stranded cDNAs were then synthesized using reverse transcriptase (Superscript II, Life Technologies) and random hexamer primers. The standard Illumina protocol was followed thereafter to develop mRNA-seq libraries. Subsequently, the library preparations were sequenced on an Illumina Hiseq platform, and $150 \mathrm{bp}$ paired-end reads were generated. All sequencing data were deposited in the NCBI and can be accessed in the Short Read Archive (SRA) under accession number SRP100344.

\section{Data processing assembly and annotation}

Raw reads in FASTQ format were processed through in-house Perl scripts. Clean reads were obtained by removing reads containing adapter, reads containing poly- $\mathrm{N}$ and low quality reads from the raw reads. At the same time, Q20, Q30, GC-content and sequence duplication level of the clean data were calculated. The sequenced left files (read1 files) from the nine samples were pooled into one big left.fq file, and right files (read2 files) into one big right.fq file. Transcriptome assembly was accomplished based on the left.fq and right.fq using Trinity (version r20140413p1) [85] with min_kmer_cov set to 2 and all other parameters set as default. The completeness of the assembly was assessed with the Benchmarking Universal Single-Copy Orthologs (BUSCO, version 3.0.2) tool [86] using the insecta_odb9 dataset. The longest transcript of each gene was defined as the 'unigene' for functional annotation. Seven databases were used to annotate the gene function: $\mathrm{Nr}$ (NCBI non-redundant protein sequences, NCBI blast 2.2.28+, E-value =1E-5); Nt (NCBI nucleotide sequences, NCBI blast 2.2.28+, E-value $=1 \mathrm{E}-5)$; Pfam (Protein family, http://pfam.xfam.org/, HMMER 3.0 package, hmmscan, E-value =0.01); KOG/COG (Clusters of Orthologous Groups of proteins, http://www.ncbi.nlm. nih.gov/COG/, NCBI blast 2.2.28+, E-value = 1E-3); Swiss Prot (a manually annotated and reviewed protein sequence database, http://www.ebi.ac.uk/uniprot/, NCBI blast 2.2.28+, E-value = 1E-5); KO (KEGG Ortholog database, http://www.genome.jp/kegg/, KAAS (version r140224), KEGG Automatic Annotation Server, E-value = 1E-10) [87]; and GO (Gene Ontology, http://www.geneon tology.org/, Blast2GO v2.5, E-value = 1E-6) [88].

\section{Analysis of gene expression patterns}

The read counts of a gene in each sample were estimated by mapping clean reads to the Trinity transcripts assembled by RSEM (version v1.2.15, bowtie2 default parameters, mismatch $=0$ ) [89]. FPKM (fragments per kilobase of transcript per million fragments mapped) was used to estimate the level of gene expression [90]. Differential gene expression analysis among conditions/groups was performed using the DEGseq $\mathrm{R}$ package (version 1.10.1) with the threshold q-value $<0.05$ and absolute Fold Change $>2$ demarcating significantly different expression levels [91]. We evaluated genetic differentiation through the Fst fixation index and different selection regimens using Tajima's D statistic. Fst and Tajima's D statistics for sliding windows were calculated using VCFtools [92], with a window of 40,000. Venn diagram analysis of DEGs between different groups was performed using the OmicShare tools, a free online platform for data analysis (http://www.omicshare.com/tools/ index.php/Home/Soft/venn). Hierarchical clustering and Venn diagram were used to illustrate the differential gene expression patterns between different groups, which were carried out using the OmicShare tools, a free online platform for data analysis (www.omicshare.com/ tools). Heatmaps were also produced using the OmicShare tools (Z-score normalization set to row, and all other parameters set as default) based on normalized FPKM data (http://www.omicshare.com/tools/Home/ Soft/heatmap).

Enrichment analysis of GO enrichment and KEGG pathway GO term enrichment analysis of DEGs was carried out using the GOseq R package (version 1.10.0) which can adjust for gene length bias in DEGs based on the Wallenius non-central hyper-geometric distribution [93]. KEGG is a database resource for understanding high-level functions 
and relationships among biological systems, such as the cell, organism, and ecosystem, from molecular-level information, which are represented by large-scale molecular datasets generated by genome sequencing and other high-throughput experimental technologies (http://www.genome.jp/kegg/). Here, KOBAS (KEGG Orthology-Based Annotation System) (version v2.0.12) [94] was used to test the statistical enrichment of DEGs in KEGG pathways.

\section{Real-time quantitative PCR analysis}

In order to validate the results from our transcriptome sequencing analysis, the relative expression levels of 15 selected genes were confirmed by reverse transcription quantitative PCR (RT-qPCR). RNA was isolated from midguts and reverse-transcribed to cDNA using PrimeScript II 1st strand cDNA synthesis kit (Takara, Dalian, China). The gene-specific primers were designed using the predicted CDSs as reference sequences. All the primers are listed in Additional file 1: Table S12. RT-qPCR reactions were prepared with the SYBR Premix Ex Taq ${ }^{\text {Tw }}$ Kit (Takara), following the manufacturer's instruction. Quantitative reactions were performed on the Real-Time PCR Detection System (ABI 7500, Applied Biosystems, USA). The qPCR cycling parameters were as follows: $95^{\circ} \mathrm{C}$ for $5 \mathrm{~min}$, followed by 40 cycles of $95^{\circ} \mathrm{C}$ for $10 \mathrm{~s}$ and $60^{\circ} \mathrm{C}$ for $30 \mathrm{~s}$, melt curves stages at $95^{\circ} \mathrm{C}$ for $15 \mathrm{~s}, 60^{\circ} \mathrm{C}$ for $1 \mathrm{~min}$, and 95 ${ }^{\circ} \mathrm{C}$ for $15 \mathrm{~s}$. Negative controls without template were included in each experiment. To check reproducibility, the qPCR reaction for each sample was performed in three technical replicates and three biological replicates. The relative gene expression was calculated using the $2^{-\Delta \Delta \mathrm{Ct}}$ method [95]. Two reference genes, Ribosomal protein L9 and Actin, were used for normalization. Data analysis was performed using the SPSS Statistics 20.0 software (IBM SPSS Statistics Inc., Chicago, IL, USA). Results are reported as mean $\pm \mathrm{SE}$.

\section{Additional files}

Additional file 1: Table S1-S3, Table S11 and Table S12. Table S1. Number of paired reads obtained by RNA-Seq. Table S2. Annotation of unigenes in different databases. Table S3. Species distribution is shown as the percentage of the total homologous sequences. Table S11. List of KEGG pathways with $P$-value $<0.05$ between the two pairwise comparisons, 'HL vs HC' and 'HL vs FX'. Table S12. Primers used in GRT-PCR (DOC $137 \mathrm{~kb}$ )

Additional file 2: Figure S1. Gene ontology classification of assembled unigenes. The 59,179 matched unigenes were classified into three functional categories: molecular function, biological process and cellular component (TIF $775 \mathrm{~kb}$ )

Additional file 3: Figure S2. KOG functional classification of all unigenes. A total of 28,896 unigenes showed significant similarity to the sequences in KOG databases and were clustered into 26 categories (TIF $754 \mathrm{~kb}$ )

Additional file 4: Figure S3. KEGG pathway distributions of midgut unigenes. The genes according to KEGG metabolic pathway involved was divided into five branches: A, Cellular processes; B, Environmental information processing; C, Genetic information processing; D, Metabolism; E, Organismal systems (TIF 1056 kb)

Additional file 5: Table S4. Primers designed for detecting simple sequence repeats (SSRs) in genes of Subpsaltria yangi (XLS $7778 \mathrm{~kb}$ )

Additional file 6: Figure S4. Cluster analysis of differentially expressed genes. Different colors indicate different levels of gene expression: from red to blue, the $\log _{10}($ FPKM +1$)$ value ranges from large to small. FX: the population occurring in Fengxiang; $\mathrm{HC}$ : the population occurring in Hancheng; HL: the population occurring in the Helan Mountains (TIF 695 kb)

Additional file 7: Table S5. Annotation of differentially expressed genes in the comparison of 'HC vS FX'. FX: the population occurring in Fengxiang; HC: the population occurring in Hancheng (XLS 286 kb)

Additional file 8: Table S6. Annotation of differentially expressed genes in the comparison of 'HL vs HC'. HC: the population occurring in Hancheng; $\mathrm{HL}$ : the population occurring in the Helan Mountains (XLS $2152 \mathrm{~kb}$ )

Additional file 9: Table S7. Annotation of differentially expressed genes in the comparison of 'HL vs FX'. FX: the population occurring in Fengxiang; $\mathrm{HL}$ : the population occurring in the Helan Mountains (XLS $895 \mathrm{~kb}$ )

Additional file 10: Table S8. Candidate DEGs related to digestion (XLS $45 \mathrm{~kb}$ )

Additional file 11: Table S9. Candidate DEGs related to detoxification and oxidation-reduction (XLS $71 \mathrm{~kb}$ )

Additional file 12: Table S10. Candidate DEGs related to stress response, water-deprivation response and putative osmoregulation (XLS $82 \mathrm{~kb}$ )

Additional file 13: Figure S5. GO enrichment of differentially expressed genes in (a) 'HL vs HC' and (b) 'HL vs FX'. The 20 most enriched GO terms are shown together with their $-\log _{2}(P$-value) and number of genes (adjacent the bars). FX: the population occurring in Fengxiang; HC: the population occurring in Hancheng; $\mathrm{HL}$ : the population occurring in the Helan Mountains (TIF $1528 \mathrm{~kb}$ )

\section{Abbreviations}

A6A8: ATPase subunits; ABC transporters: ATP-binding cassette transporters: AQP: Aquaporin; BP: Biological process; BUSCO: Benchmarking universal single-copy orthologs; CAT: Catalase; CC: Cellular component;

CDNA: Complementary DNA; CDS: Coding sequence; CE: Carboxylesterases; COI: Cytochrome c oxidase subunit l; COIl: Cytochrome c oxidase subunit II; Cytb: Cytochrome b; DEGs: Differentially expressed genes;

DEPC: Diethylpyrocarbonate; DNA: Deoxyribonucleic acid; FC: Fold change; FPKM: Fragments per kilobase of transcript per million fragments mapped; Fst: Fixation index; FX: Fengxiang; GO: Gene Ontology; GPX: Glutathione peroxidase; GST: Glutathione S-transferase; HC: Hancheng; HL: Helan Mountains; Hsp: Heat shock proteins; KAAS: KEGG automatic annotation server; KEGG: Kyoto encyclopedia of genes and genomes; KO: KEGG ortholog database; KOBAS: KEGG orthology-based annotation system; KOG: Eukaryotic orthologous groups; NCBI: National center for biotechnology information; $\mathrm{Nr}$ : NCBI non-redundant protein sequences; Nt: NCBI nucleotide sequences; P450: Cytochrome P450 monooxygenases; PBS: Phosphate buffered saline; PCR: Polymerase chain reaction; Pfam: Protein family; RNA: Ribonucleic acid; RNA-Seq: RNA sequencing; ROS: Reactive oxygen species; RT-qPCR: Real-time quantitative PCR; Slc: Solute carriers; SOD: Superoxide dismutase; SPSS: Statistical package for the social sciences; SRA: Short read archive; SSR: Simple sequence repeats; TCA cycle: Tricarboxylic acid cycle; tRNA: Transfer RNA; UGT: UDP-glycosyltransferases

\section{Acknowledgments}

We would like to express our deep gratitude to Dandan Wang (Northwest A\&F University, China) for her help with specimen dissection. We thank Yunxiang Liu, Changqing Luo, Mengmeng Qi, Zhenpeng Wang, and Zhi Huang (Northwest A\&F University, China) for their help with specimen collection. 


\section{Funding}

This work was supported by the National Natural Science Foundation of China (Grant No. 31572302, No. 31772505) to C.W. The authors declare there are no competing financial interests. The funders had no role in the study design, data collection and analysis, interpretation of data, or writing of the manuscript.

\section{Availability of data and materials}

The sequence data (SRP100344) is available at https://www.ncbi.nlm.nih.gov/ sra/SRP100344.

\section{Authors' contributions}

$\mathrm{CW}$ and $\mathrm{ZH}$ designed the study. $\mathrm{ZH}$ performed the experiments. $\mathrm{ZH}$ and $\mathrm{CW}$ analysed the data. $\mathrm{ZH}$ and $\mathrm{CW}$ wrote the paper. Both authors read and approved the final manuscript.

\section{Ethics approval and consent to participate}

This study was carried out in full compliance with the laws of the People's Republic of China. No specific permits were required for our field investigation. The study species is not included in the 'List of Protected Animals in China'.

\section{Consent for publication}

Not applicable.

\section{Competing interests}

The authors declare that they have no competing interests.

\section{Publisher's Note}

Springer Nature remains neutral with regard to jurisdictional claims in published maps and institutional affiliations.

Received: 18 November 2018 Accepted: 20 February 2019

Published online: 07 March 2019

\section{References}

1. Cronin JT, Abrahamson WG. Do parasitoids diversify in response to hostplant shifts by herbivorous insects. Ecol Entomol. 2001;26:347-55.

2. Vogel H, Musser RO, Celorio-Mancera M. Transcriptome responses in herbivorous insects towards host plant and toxin feeding. Annu Plant Rev. 2014:47:197-233.

3. Tilmon KJ. Specialization, speciation, and radiation: the evolutionary biology of herbivorous insects. Berkeley: University of California Press; 2008.

4. Chen KF. New genera and species of Chinese cicadas with synonymical and nomenclatorial notes. J New York Entomol Soc. 1943:51:19-52.

5. Chou I, Lei Z, Li L, Lu X, Yao W. The Cicadidae of China (Homoptera: Cicadoidea). Hong Kong: Tianze Press; 1997.

6. Marshall DC, Moulds M, Hill KBR, Price BW, Wade EJ, Owen CL, et al. A molecular phylogeny of the cicadas (Hemiptera: Cicadidae) with a review of tribe and subfamily level classification. Zootaxa. 2018;4424:1-64.

7. Luo C, Wei C. Intraspecific sexual mimicry for finding females in a cicada: males produce 'female sounds' to gain reproductive benefit. Anim Behav. 2015;102:69-76.

8. Liu YX, Dietrich $\mathrm{CH}$, Wei C. Genetic divergence, population differentiation and phylogeography of the cicada Subpsaltria yangi based on molecular and acoustic data: an example of the early stage of speciation? BMC Evol Biol. 2019;19:5

9. Hoang K, Matzkin LM, Bono JM. Transcriptional variation associated with cactus host plant adaptation in Drosophila mettleri populations. Mol Ecol. 2015;24:5186-99.

10. Zhong H, Li F, Chen J, Zhang J, Li F. Comparative transcriptome analysis reveals host-associated differentiation in Chilo suppressalis (Lepidoptera: (rambidae). Sci Rep. 2017;7:13778.

11. Matzkin LM. Population transcriptomics of cactus host shifts in Drosophila mojavensis. Mol Ecol. 2012;21:2428-39.

12. De Panis DN, Padró J, Furió-Tarí P, Tarazona S, Milla Carmona PS, Soto IM, et al. Transcriptome modulation during host shift is driven by secondary metabolites in desert Drosophila. Mol Ecol. 2016;25:4534-50.

13. Williams KS, Simon C. The ecology, behavior and evolution of periodical cicadas. Ann Rev Ecol System. 1995;40:269-95.
14. Hou Z, Li Q, Yang M, Liu Y, Wei C. Ecology of Meimuna mongolica (Hemiptera: Cicadidae) nymphs: instars, morphological variation, vertical distribution and population density, host-plant selection, and emergence phenology. J Insect Sci. 2015:15:42.

15. Johnston MC. The species of Ziziphus indigenous to United States and Mexico. Am J Bot. 1963:50:1020-7.

16. Che $Y$, Zheng $B Q$, Teng $Y R$, Zhang $Y Q$. Chemical constituents from the leaves of Ziziphus jujuba var. spinosa. Chinese Traditional Patent Medicine. 2012;34:686-8.

17. Zhang QQ, Qi W, Wang WN, Li MX, Dang F, Liu XQ. Isolation and identification of chemical constituents from Ziziphi spinosae branch and folium. J Shenyang Pharm Univ. 2013;30:917-20+32.

18. Dewick MP. Medicinal natural products: a biosynthetic approach. 2 nd ed. West Sussex: John Wiley and Sons; 2002.

19. Jiang H, Li W, Li S, Shi L, Gao Y, Wu J. Relationship analysis of five species in the genus Ephedra L. by RAPD. J Gansu Agric Univ. 2006;41:49-52.

20. Reti L, Castrillón JA. Cactus alkaloids. I. Trichocereus terscheckii (Parmentier) Britton and Rose. J Am Chem Soc. 1951;73:1767-9.

21. Ogunbodede O, McCombs D, Trout K, Daley P, Terry M. New mescaline concentrations from 14 taxa/cultivars of Echinopsis spp. (Cactaceae) ("San Pedro") and their relevance to shamanic practice. J Ethnopharmacol. 2010; 131:356-62.

22. Fanara JJ, Fontdevila A, Hasson E. Oviposition preference and life history traits in cactophilic Drosophila koepferae and D. buzzatii in association with their natural hosts. Evol Ecol. 1999;13:173-90.

23. Fanara JJ, Hasson E. Oviposition acceptance and fecundity schedule in the cactophilic sibling species Drosophila buzzatii and D. koepferae on their natural hosts. Evolution. 2001:55:2615-9.

24. Fanara JJ, Folguera G, Iriarte PF, Mensch J, Hasson E. Genotype by environment interactions in viability and developmental time in populations of cactophilic Drosophila. J Evolution Biol. 2006;19:900-8.

25. Soto EM, Goenaga J, Hurtado JP, Hasson E. Oviposition and performance in natural hosts in cactophilic Drosophila. Evol Ecol. 2012;26:975-90.

26. Futuyma DJ, Peterson SC. Genetic variation in the use of resources by insects. Annu Rev Entomol. 1985;30:217-38.

27. Rossiter MC. Genetic and phenotypic variation in diet breadth in a generalist herbivore. Evol Ecol. 1987;1:272-82.

28. Qi M, Wei S, Wei C. Identification of candidate olfactory genes in cicada Subpsaltria yangi by antennal transcriptome analysis. Comp Biochem Physiol Part D Genomics Proteomics. 2018;28:122-33.

29. Dahanukar A, Hallem EA, Carlson JR. Insect chemoreception. Curr Opin Neurobiol. 2005;15:423.

30. Xue W, Jia F, Yong Z, Xu Q, Han Z, Sun J, et al. Identification and expression analysis of candidate odorant-binding protein and chemosensory protein genes by antennal transcriptome of Sitobion avenae. PLoS One. 2016:11:e0161839.

31. Wang P, Granados RR. Molecular structure of the peritrophic membrane (PM): identification of potential PM target sites for insect control. Arch Insect Biochem Physiol. 2001;47:110-8.

32. Hakim RS, Baldwin K, Smagghe G. Regulation of midgut growth, development, and metamorphosis. Annu Rev Entomol. 2010;55:593.

33. Celorio-Mancera M, Wheat CW, Vogel H, Söderlind L, Janz N, Nylin S. Mechanisms of macroevolution: polyphagous plasticity in butterfly larvae revealed by RNA-Seq. Mol Ecol. 2013:22:4884-95.

34. Ragland GJ, Almskaar K, Vertacnik KL, Gough HM, Feder JL, Hahn DA, et al. Differences in performance and transcriptome-wide gene expression associated with Rhagoletis (Diptera: Tephritidae) larvae feeding in alternate host fruit environments. Mol Ecol. 2015:24:2759-76.

35. Chikate YR, Tamhane VA, Joshi RS, Gupta VS, Giri AP. Differential protease activity augments polyphagy in Helicoverpa armigera. Insect Mol Biol. 2013; 22:258-72.

36. Grbić M, Van Leeuwen $T$, Clark RM, Rombauts $S$, Rouzé $P$, Grbić V, et al. The genome of Tetranychus urticae reveals herbivorous pest adaptations. Nature. 2011;479:487-92.

37. Sun $\mathrm{H}, \mathrm{Pu} J$, Chen F, Wang J, Han Z. Multiple ATP-binding cassette transporters are involved in insecticide resistance in the small brown planthopper, Laodelphax striatellus. Insect Mol Biol. 2017;26:343-55.

38. Ren J, Chung-Davidson YW, Yeh CY, Scott C, Brown T, Li W. Genome-wide analysis of the ATP-binding cassette (ABC) transporter gene family in sea lamprey and Japanese lamprey. BMC Genomics. 2015;16:436.

39. Shanker M, Willcutts $D$, Roth JA, Ramesh $R$. Drug resistance in lung cancer. Lung Cancer. 2010;1:23-36. 
40. Srinivas R, Udikeri SS, Jayalakshmi SK, Sreeramulu K. Identification of factors responsible for insecticide resistance in Helicoverpa armigera. Comp Biochem Phys C. 2004;137:261-9.

41. Zibaee A, Sendi JJ, Ghadamyari M, Alinia F, Etebari K. Diazinon resistance in different selected strains of Chilo suppressalis (Lepidoptera: Crambidae) in northern Iran. J Econ Entomol. 2009;102:1189-96.

42. Halliwell B. Oxygen and nitrogen are pro-carcinogens. Damage to DNA by reactive oxygen, chlorine and nitrogen species: measurement, mechanism and the effect of nutrition. Mutat Res. 1999;443:37-52.

43. Ahmad S, Pardini RS. Mechanisms for regulating oxygen toxicity in phytophagous insects. Free Radic Biol Med. 1990;8:401-13.

44. Mittapalli O, Neal JJ, Shukle RH. Antioxidant defense response in a galling insect. Proc Natl Acad Sci U S A. 2007;104:1889-94.

45. George DGM, Gatehouse AMR. Oxidative stress enzymes in Busseola fusca. Int J Curr Microbiol App Sci. 2013;2:485-95.

46. Barbehenn RV. Gut-based antioxidant enzymes in a polyphagous and a graminivorous grasshopper. J Chem Ecol. 2002;28:1329-47.

47. Zhu X, Xie S, Armengaud J, Xie W, Guo Z, Kang S, et al. Tissue-specific proteogenomic analysis of Plutella xylostella larval midgut using a multialgorithm pipeline. Mol Cell Proteomics. 2016;15:1791-807.

48. Goto SG, Kimura MT. Heat- and cold-shock responses and temperature adaptations in subtropical and temperate species of Drosophila. J Insect Physiol. 1998:44:1233-9.

49. Sørensen JG, Kristensen TN, Loeschcke V. The evolutionary and ecological role of heat shock proteins. Ecol Lett. 2003;6:1025-37.

50. Feder JH, Rossi JM, Solomon J, Solomon N, Lindquist S. The consequences of expressing hsp70 in Drosophila cells at normal temperatures. Genes Dev. 1992;6:1402-13.

51. Krebs RA, Loeschcke V. Costs and benefits of activation of the heat-shock response in Drosophila melanogaster. Funct Ecol. 1994;8:730-7.

52. Coleman JS, Heckathorn SA, Hallberg RL. Heat-shock proteins and thermotolerance: linking molecular and ecological perspectives. Trends Ecol Evol. 1995;10:305-6.

53. Sanders BM, Hope C, Pascoe VM, Martin LS. Characterization of the stress protein response in two species of Collisella limpets with different temperature tolerances. Physiol Zool. 1991;64:1471-89.

54. Bosch TC, Krylow SM, Bode HR, Steele RE. Thermotolerance and synthesis of heat shock proteins: these responses are present in Hydra attenuata but absent in Hydra oligactis. Proc Natl Acad Sci U S A. 1988;85:7927-31.

55. Walsberg G. Small mammals in hot deserts: some generalizations revisited. Bioscience. 2000;50:109-20.

56. MacManes MD. Severe acute dehydration in a desert rodent elicits a transcriptional response that effectively prevents kidney injury. Am J Physiol Renal Physiol. 2017;313:F262-72.

57. Heymann J, Engel A. Aquaporins: phylogeny, structure, and physiology of water channels. News Physiol Sci. 1999;14:187-93.

58. Brown D, Katsura T, Kawashima M, Verkman AS, Sabolic I. Cellular distribution of the aquaporins: a family of water channel proteins. Histochem Cell Biol. 1995;104:1-9.

59. Ma T, Yang B, Gillespie A, Carlson EJ, Epstein CJ, Verkman AS. Severely impaired urinary concentrating ability in transgenic mice lacking aquaporin1 water channels. J Biol Chem. 1998;273:4296-9.

60. Verkman AS. Physiological importance of aquaporin water channels. Ann Med. 2002;34:192-200

61. Gomes D, Agasse A, Thiébaud P, Delrot S, Gerós H, Chaumont F. Aquaporins are multifunctional water and solute transporters highly divergent in living organisms. Biochim Biophys Acta. 1788;2009:1213-28.

62. Ishibashi K, Hara S, Kondo S. Aquaporin water channels in mammals. Clin Exp Nephrol. 2009;13:107-17.

63. Fenton RA, Knepper MA. Mouse models and the urinary concentrating mechanism in the new millennium. Physiol Rev. 2007;87:1083-112.

64. Huang Y, Tracy R, Walsberg GE, Makkinje A, Fang P, Brown D, et al. Absence of aquaporin- 4 water channels from kidneys of the desert rodent Dipodomys merriami merriami. Am J Physiol Renal Physiol. 2001;280:F794-802.

65. Wu H, Guang X, Al-Fageeh MB, Cao J, Pan S, Zhou H, et al. Camelid genomes reveal evolution and adaptation to desert environments. Nat Commun. 2014:5:5188

66. Hediger MA, Romero MF, Peng JB, Rolfs A, Takanaga H, Bruford EA. The ABCs of solute carriers: physiological, pathological and therapeutic implications of human membrane transport proteins. Pflugers Arch. 2004; 447:465-8.
67. He L, Vasiliou K, Nebert DW. Analysis and update of the human solute carrier (SLC) gene superfamily. Hum Genomics. 2009;3:195-205.

68. Marra NJ, Eo SH, Hale MC, Waser PM, DeWoody JA. A priori and a posteriori approaches for finding genes of evolutionary interest in non-model species: osmoregulatory genes in the kidney transcriptome of the desert rodent Dipodomys spectabilis (banner-tailed kangaroo rat). Comp Biochem Physiol Part D Genomics Proteomics. 2012;7:328-39.

69. MacManes MD, Eisen MB. Characterization of the transcriptome, nucleotide sequence polymorphism, and natural selection in the desert adapted mouse Peromyscus eremicus. PeerJ. 2014;2:e642.

70. Kordonowy L, MacManes M. Characterizing the reproductive transcriptomic correlates of acute dehydration in males in the desert-adapted rodent, Peromyscus eremicus. BMC Genomics. 2017;18:473.

71. Giorello FM, Feijoo M, D'Elía G, Naya DE, Valdez L, Opazo JC, et al. An association between differential expression and genetic divergence in the Patagonian olive mouse (Abrothrix olivacea). Mol Ecol. 2018;27:3274-86.

72. Janz N, Nylin S, Wahlberg N. Diversity begets diversity: host expansions and the diversification of plant-feeding insects. BMC Evol Biol. 2006;6:4.

73. Crispo E. The Baldwin effect and genetic assimilation: revisiting two mechanisms of evolutionary change mediated by phenotypic plasticity. Evolution. 2007:61:2469-79.

74. Gu L, Liu Y, Que P, Zhang Z. Quaternary climate and environmental changes have shaped genetic differentiation in Chinese pheasant endemic to the eastern margin of the Qinghai-Tibetan plateau. Mol Phylogenet Evol. 2013;67:129-39.

75. Cooley JR, Simon C, Marshall DC. Temporal separation and speciation in periodical cicadas. BioScience. 2003;53:151-7.

76. MacMillan HA, Knee JM, Dennis AB, Udaka H, Marshall KE, Merritt TJS, et al. Cold acclimation wholly reorganizes the Drosophila melanogaster transcriptome and metabolome. Sci Rep. 2016;6:28999.

77. Des Marteaux LE, McKinnon AH, Udaka H, Toxopeus J, Sinclair BJ. Effects of cold-acclimation on gene expression in fall field cricket (Gryllus pennsylvanicus) ionoregulatory tissues. BMC Genomics. 2017;18:357.

78. Huang HJ, Xue J, Zhuo JC, Cheng RL, Xu HJ, Zhang CX. Comparative analysis of the transcriptional responses to low and high temperatures in three rice planthopper species. Mol Ecol. 2017;26:2726-37.

79. Larsson E, Tremaroli V, Lee YS, Koren O, Nookaew I, Fricker A, et al. Analysis of gut microbial regulation of host gene expression along the length of the gut and regulation of gut microbial ecology through MyD88. Gut. 2012;61: 1124-31.

80. Camp JG, Frank CL, Lickwar CR, Guturu H, Rube T, Wenger AM, et al. Microbiota modulate transcription in the intestinal epithelium without remodeling the accessible chromatin landscape. Genome Res. 2014;24: 1504-16.

81. Sommer F, Nookaew I, Sommer N, Fogelstrand P, Bäckhed F. Site-specific programming of the host epithelial transcriptome by the gut microbiota. Genome Biol. 2015;16:62.

82. Pan WH, Sommer F, Falk-Paulsen M, Ulas T, Best P, Fazio A, et al. Exposure to the gut microbiota drives distinct methylome and transcriptome changes in intestinal epithelial cells during postnatal development. Genome Med. 2018;10:27.

83. Beamer RH. Studies on the biology of Kansas Cicadidae. The University of Kansas Science Bulletin. 1928;18:155-230.

84. Karban R, Black CA, Weinbaum SA. How 17-year cicadas keep track of time. Ecol Lett. 2000;3:253-6.

85. Grabherr MG, Haas BJ, Yassour M, Levin JZ, Thompson DA, Amit I, et al. Fulllength transcriptome assembly from RNA-Seq data without a reference genome. Nat Biotechnol. 2011;29:644-52.

86. Simão FA, Waterhouse RM, loannidis P, Kriventseva EV, Zdobnov EM. BUSCO: assessing genome assembly and annotation completeness with single-copy orthologs. Bioinformatics. 2015;31:3210-2.

87. Kanehisa M, Araki M, Goto S, Hattori M, Hirakawa M, Itoh M, et al. KEGG for linking genomes to life and the environment. Nucleic Acids Res. 2008;36: D480-4.

88. Götz S, García-Gómez JM, Terol J, Williams TD, Nagaraj SH, Nueda MJ, et al. High-throughput functional annotation and data mining with the Blast2GO suite. Nucleic Acids Res. 2008;36:3420-35.

89. Li B, Dewey C. RSEM: accurate transcript quantification from RNA-Seq data with or without a reference genome. BMC Bioinformatics. 2011;12:323.

90. Trapnell C, Williams B, Pertea G, Mortazavi A, Kwan G, van Baren M, et al Transcript assembly and quantification by RNA-Seq reveals unannotated 
transcripts and isoform switching during cell differentiation. Nat Biotech. 2010;28:511-5

91. Wang L, Feng Z, Wang X, Wang X, Zhang X. DEGseq: an R package for identifying differentially expressed genes from RNA-seq data. Bioinformatics. 2010;26:136-8

92. Danecek P, Auton A, Abecasis G, Albers CA, Banks E, DePristo MA, et al. The variant call format and VCFtools. Bioinformatics. 2011;2715:2156-8.

93. Young MD, Wakefield MJ, Smyth GK, Oshlack A. Gene ontology analysis for RNA-seq: accounting for selection bias. Genome Biol. 2010;11:R14.

94. Mao X, Cai T, Olyarchuk JG, Wei L. Automated genome annotation and pathway identification using the KEGG Orthology (KO) as a controlled vocabulary. Bioinformatics. 2005;21:3787-93.

95. Livak KJ, Schmittgen TD. Analysis of relative gene expression data using real-time quantitative PCR and the $2^{-\Delta \Delta T}$ method. Methods. 2001;25:402-8.

\section{Ready to submit your research? Choose BMC and benefit from:}

- fast, convenient online submission

- thorough peer review by experienced researchers in your field

- rapid publication on acceptance

- support for research data, including large and complex data types

- gold Open Access which fosters wider collaboration and increased citations

- maximum visibility for your research: over $100 \mathrm{M}$ website views per year

At BMC, research is always in progress.

Learn more biomedcentral.com/submissions 